Mike Geppert/ Dirk Matten/ Peggy Schmidt

\title{
Die Bedeutung institutionalistischer Ansätze für das Verständnis von Organisations- und Managementprozessen in multinationalen
}

\section{Unternehmen}

Es ist auffällig, dass sich die sozialwissenschaftlich orientierte Organisationsforschung nur sehr wenig und theoretisch recht einseitig mit der MNU als Organisation beschäftigt. Organisationstheoretische Erklärungsmodelle, welche das Verhalten in und von Organisationen jenseits technischer und ökonomischer Unternehmensumwelten erklären, sind daher eher rar und erst seit kurzem in einschlägigen Forschungsarbeiten zu finden. Das Ziel dieses Beitrages ist, die jüngere und vorwiegend englischsprachige Diskussion über den Einfluß von Institutionen und unterschiedlichen lokalen Rationalitäten auf Organisations- und Managementprozesse in MNU aufzuarbeiten. Im folgenden werden die Grundlagen, wesentliche Ansätze, Kernaussagen und Konsequenzen des europäischen und amerikanischen Institutionalismus dargestellt. Insbesondere wird der Beitrag die Bedeutung aber auch Grenzen dieser beiden Ansätze für die Analyse von Veränderungsprozessen in MNU skizzieren und evaluieren. Im Vergleich zum noch dominanten ,evolutionären Paradigma“ in der internationalen Managementforschung wird hier das alternative Erklärungspotential von sozialwissenschaftlichen Institutionentheorien herausgestellt und anhand vier verschiedener Aspekten diskutiert: dem Einfluss des Heimatlandes, der Macht und Autonomie von Tochtergesellschaften, sowie der Entstehung transnationaler sozialer Räume. 


\section{Ausgangspunkt und Problemstellung}

Die Analyse von MNU als Organisationen hat bisher nur sehr wenig Interesse von soziologisch orientierten Organisationsforschern gefunden (Rosenzweig/Singh, 1991; Westney/Zaheer, 2001). Studien zum Verhalten von und in MNU werden immer noch sehr stark von ökonomisch rationalen (Buckley, 1996; Casson, 1997; Buckley/Ghauri, 1999) bzw. kontingenztheoretisch funktionalen (wie z.B. Bartlett, Doz, Egelhoff, Ghoshal, Prahalad) Annahmen bestimmt. Dennoch gibt es seit Beginn der 90er Jahre insbesondere in Nordamerika aber auch in Großbritannien eine anwachsende Zahl von Forschungsarbeiten, die ausgehend von einer organisationssoziologischen Kritik bisherig dominanter kontingenztheoretischen Studien, die Rolle von institutionellen Umwelten für die Organisation und das Management von MNU betonen.

Dieser Aufsatz diskutiert den Einfluß unterschiedlicher kultureller and institutioneller Einflüsse auf das Management und die Organisation von MNU. Ausgehend von einer kritischen Rezeption kontingenztheoretisch sowie kulturalistisch orientiertrer Studien von Organisation und Management in MNU wird ausgiebig auf die Bedeutung institutionalistischer Erklärungsansätze für Verständnis organisatorischer Entwicklungstendenzen in MNU eingegangen. Besondere Aufmerksamkeit werden wir zuerst auf den Beitrag des sogenannten europäischen Institutionalismus richten. Anschließend setzen wir uns damit auseinander, inwieweit Kernargumente amerikanischer Institutionalisten gewinnbringend zum besseren Verständnis sozialer Praktiken und organisationaler Prozesse in MNU beitragen können. In einer zusammenführenden Diskussion beider Ansätze, basierend auf einschlägigen empirischer Untersuchungen und auch Befunden aus eigener Forschung, wird die relevanz dieser soziologischen Ansätze für die Organisationsforschung im Bereich der MNU herausgearbeitet.

\section{Die Grundannahmen und Kritik kontingenztheoretischen Annahmen}

Die gegenwärtig einflussreichsten Arbeiten zum Management von MNU im anglosächsischen Sprachraum beruhen weitestgehend noch auf kontingenztheoretischen Grundannahmen. Diese Richtung, eng verbunden mit dem klassischen Ansatz der strategischen Unternehmensführung (Porter, Ansoff), fokussiert auf die 
Managementstrukturen von MNU und analysiert die Beziehung zur Umwelt in eher technischen und ökonomischen Kategorien. Der bekannteste und auch tonangebende Beitrag dieser Richtung ist das „evolutionäre Paradigma“ von Bartlett und Ghoshal (Bartlett/Ghoshal, 1989; 1997). Grundannahme dieses Ansatzes ist, dass sich trotz unterschiedlicher Aufgabenumwelten im Internationalisierungsprozess idealtypische Formen von ähnlichen Organisationsstrukturen herausbilden. Die Typologie mündet in der evolutionären Endstufe der „transnationalen Organisation“, deren Strategie und Struktur als am flexibelsten und effizientesten für die Lösung des global-lokal Paradoxes angesehen werden. Hiermit wird ein neuer „one best way“ für die Organisation der global erfolgreichen MNU vorgeschlagen. Allerdings gibt es bisher nur wenig empirische Belege für die Existenz einer anwachsenden Zahl erfolgreich agierender „transnationaler“ Unternehmen (z.B. Belanger et al., 1999).

Insbesondere für die internationale Managementforschung ist daher der Kritik von Scott (1998) durchaus zu zustimmen, dass sich organisationstheoretische Forschung lange Zeit hauptsächlich auf den Einfluss technischer und ökonomischer Umwelten auf Unternehmenshandeln konzentriert hat und dabei die Bedeutung institutioneller Umwelten und ihrer symbolischen kulturellen und kognitiven Effekte vernachlässigt hat. Die organisationssoziologische Kritik fokussiert im wesentlichen auf die Feststellung, die Sicht auf Akteure in MNU als rational Handelnde, welche Ihre Entscheidungen an einem am Eigeninteresse ausgerichteten ökonomischen KostenNutzen Kalkül ausrichten, würde weitaus zu kurz greifen. Insbesondere vernachlässigen diese Ansätze die soziale Einbettung rationaler Entscheidungsprozesse (Granovetter, 1992), Irrationalität und Unsicherheit in Organisationen (Brunsson, 1985), sowie die soziale Konstruktion von Märkten und unterschiedlichen lokalen Rationalitäten in den MNU (Morgan, 2001).

Kritik an universellen Konvergenzannahmen in der internationalen Managementforschung und der Rolle von MNU in diesem Prozess kommt vor allem von sogenannten „High context“ Ansätzen in der international vergleichenden Managementforschung. ${ }^{1}$ Im Unterschied zu universalistischen Theorien sind „High context“ Ansätze eher historisch, empirisch und komparativ orientiert. Ländervergleichende Studien wiederlegen die Konvergenzannahmen ersterer Ansätze und betonen die kulturelle und institutionelle Vielfalt von Organisations- und Managementformen in unterschiedlichen nationalen Kontexten. So kamen z.B. Child und Kieser bereits vor mehr als 20 Jahren in einer britisch-deutschen Vergleichsstudie 
zu dem Ergebnis, dass neben situativen Faktoren auch nationalkulturelle Faktoren Managementrollen und -strukturen in Firmen beeinflussen (Child/Kieser, 1979). Im folgenden werden wir zunächst kurz auf die Kernargumente kulturalistischer Ansätze in der international vergleichenden Managementforschung eingehen, um uns dann eingehend mit den Ergebnissen institutionalistischen Untersuchungen auseinander zusetzen.

\section{Bedeutung und Grenzen von kulturalistische Ansätzen}

Insbesondere verbunden mit den Namen Hofstede und Trompenaars hat sich bereits recht früh ein Strang in der vergleichenden internationalen Organisationsforschung herausgebildet, welche den Einfluß von unterschiedlichen Nationalkulturen auf lokale Organisationen und deren Management untersucht hat. Der Kernpunkt dieser Ansätze besteht in der Annahme, dass kulturelle Grundannahmen, Werte und Normen relativ überdauernd in Gesellschaften eingebettet sind und dass diese Formen des „,collective programming of the mind“" von Gesellschaft zu Gesellschaft fundamental variieren (Hampden-Turner/Trompenaars, 1993; Hofstede, 1994; ders., 2001; HampdenTurner/Trompenaars, 2000). Dieser theoretische Zugang, oftmals als „ideationaler Ansatz“ von Organisationskultur bezeichnet (Child, 1981), behandelt Kultur im wesentlichen als basierend auf in der primären Sozialisation erworbenen kulturellen Programmen einer spezifischen Nationalkultur. Wir verweisen im vorliegenden Kontext auf die Literatur, die sich kritisch mit Hofstede auseinandergesetzt hat (Sorge, 1989; Schmidt, 2001; McSweeney, 2002).

Während Hofstedes Verdienst eindeutig in der Überwindung von ökonomistischen Verengungen besteht, fordern Autoren wie Child (1981) einen mehr „adaptiven“ Kulturbegriff. Dieser berücksichtigt insbesondere auch die Tatsache, dass Kulturen nicht allein auf einer abstrakten Werte- und Normenebene in Gesellschaften existieren. Sie können letztlich nur insoweit Gegenstand sozialwissenschaftlicher Forschung werden, wie sie sich in konkreten sozialen Praktiken und gesellschaftlichen Institutionen manifestieren. Somit steht der im folgenden eingehender diskutierte Ansatz zwar nicht direkt im Gegensatz zum Begriff der Nationalkultur. Er geht allerdings in der Sichtweise über die reine Wert- und Normenebene deutlich hinaus. 


\section{Institutionalistische Ansätze in der international vergleichenden Managementforschung}

Institutionalistische Ansätze sehen MNU als soziale Akteure, welche zutiefst in den jeweiligen sozialen, kulturellen, politischen und ökonomischen Kontext ihres Umfeldes eingebettet sind. Zentrales Interesse institutionalistischer Erklärungsmodelle ist die Forschungsfrage, wie sich Organisationen an ihre institutionellen Umwelten anpassen.

Grundlegend für das Verständnis und die Anwendung institutionalistischer Ansätze in der Literatur zum internationalen Management ist die Differenzierung zwischen europäischem und amerikanischen Institutionalismus (Tempel/Walgenbach, 2003) ${ }^{2}$. Der wesentliche Unterschied besteht darin, dass ersterer prinzipiell die Verschiedenartigkeit nationaler Kulturen und „national business systems“ (im folgenden NBS) hervorhebt während letzterer den Schwerpunkt auf die globale Diffusion von Praktiken, Strukturen und Institutionen legt. Der zentrale Transmissionsmechanismus des europäischen Institutionalismus besteht in den Institutionen des jeweiligen „national business systems“ (NBS). Internationale Vergleichstudien zeigen, dass nationale Unterschiede von betrieblichen Struktur- und Handlungsmustern nicht hinreichend durch die Aufgabenumwelt oder ökonomische Logik des Marktes erklärt werden können, sondern im wesentlichen durch ihre institutionelle Einbettung in sehr unterschiedliche „societal contexts“ (Sorge, 1991), „industrial orders“ (Lane, 1994), „social systems of production”

(Hollingsworth/Boyer, 1997) oder eben „national business systems“ (Whitley, 1997) bestimmt werden. Als solche ist diese Theoriefamilie nicht in erster Linie auf die MNU als Untersuchungsobjekt fokussiert, erfährt aber in jüngster Zeit gerade aus dieser Richtung verstärkte Aufmerksamkeit (Morgan/Kristensen/Whitley, 2001; Djelic/Quack, 2003). Im Gegensatz dazu betont jedoch der amerikanische Institutionalismus, dass Organisationen im selben „organisationalen Feld“ (z.B. im industriellen Sektor) über nationalstaatliche Grenzen hinweg ähnliche kulturelle Muster, Managementkonzepte und -strukturen entwickeln (Meyer/Rowan, 1977; DiMaggio/Powell, 1983). Damit wird z.B. die verstärkte globale Ausbreitung des Shareholder Value-Denkens oder der ISO 9000 Konzepte in weltweit operierenden Unternehmen erklärt (Meyer, 2000; Walgenbach, 2000). Somit knüpft der 
phänomenologische Institutionalismus an die universelle Logik oben diskutierter Theorien der MNU an.

\subsection{Der europäische Institutionalismus}

Als der am weitesten und theoretisch umfassendste Ansatz im europäischen Institutionalismus kann der „National Business Systems“ Ansatz von Richard Whitley angesehen werden (Whitley, 1992; Whitley/Kristensen, 1996; Whitley, 1997; Whitley/Kristensen, 1997; Quack/Morgan/Whitley, 1999; Whitley, 1999; Morgan/Kristensen/Whitley, 2001).

\section{Abbildung 1 etwa hier}

Ausgangspunkt des theoretischen Bezugsrahmens im NBS-Ansatz (siehe Abb. 1) sind die Schlüsselcharakteristika des historisch gewachsenen Institutionengefüges eines Landes, i.d.R. synonym mit einem Nationalstaat. Hier unterscheidet Whitley vier Kernelemente, das politische System, das Finanzsystem, die Beschäftigungs- und Bildungspolitik („Labour system“) sowie das Kulturelle System. Abhängig von den institutionellen Rahmenbedingungen einer Volkswirtschaft bildet sich dann ein konkretes NBS heraus (für Details sei auf Abb. 1 sowie die zitierte Literatur verwiesen).

In seiner jüngeren Geschichte hat der Ansatz insbesondere dadurch Auftrieb erhalten, dass nach Beendigung der meisten (sozialistischen) Planwirtschaftsmodelle bei gleichzeitiger Intensität von Globalisierungsprozessen sich verstärkt das Bewusstsein dafür durchsetzt, dass innerhalb des kapitalistischen Wirtschaftsmodells erhebliche Unterschiede und teils geradezu „divergente“ Kapitalismusentwürfe ${ }^{3}$ vorliegen. Dabei hat sich eine Vielzahl von Forschern, allen voran die sog. „Aix-Group“ in Aix-enProvence um Maurice und Sorge, der Frage gewidmet, wie auf relativ engem Raum in Europa vergleichsweise sehr unterschiedliche Industriestrukturen, betriebliche Qualifikations- und Mitbestimmungspotentiale sowie Führungsparadigmen entwickelt haben (Maurice/Sorge, 2000), wobei deutsch-britische Vergleichstudien einen relativ 
breiten Raum einnehmen (Child/Kieser, 1979; Maurice, 1980; Sorge/Warner, 1986; Lane, 1992; 1994; 2000; 2001; Geppert/Matten/Williams, 2002).

\subsection{Der amerikanische Institutionalismus}

Im Gegensatz zum europäischen Institutionalismus liegt der Fokus der Vertreter des amerikanischen Institutionalismus auf der Homogenisierung von institutionellen Umwelten über nationale Grenzen hinweg In Anlehnung an Pionierarbeiten in der Organisationstheorie von Weber (1905) über Selznick (1949) bis hin zu Berger und Luckmann (Berger/Luckmann, 1980) wird davon ausgegangen, dass regulative, normative und kognitive Mechanismen dafür sorgen, dass Organisationen „rationalized organizational practices“ bzw. „,best practices“ übernehmen. Dies geschieht nicht, weil sie effizienter, profitabler oder transaktionskosten-optimal sind, sondern weil sie in bestimmten institutionellen Umwelten als legitim angesehen werden (Scott, 1998). Die Idee der Legitimität von Handeln in bestimmten „organizational fields“ (DiMaggio/Powell, 1983: 64-65), wie z.B. industriellen Sektoren, ist damit nicht mehr national definiert, sondern wird als global oder transnational begriffen und knüpft damit direkt an die aktuelle Diskussion um die Rolle von MNU in diesem Prozess an.

Ein wichtiges theoretisches Konstrukt zur Erklärung der weltweiten Annäherung von Organisationen, welche im selben „organizational field“ operieren, ist die Idee des Isomorphismus. Unterschieden werden dabei drei Formen (DiMaggio/Powell, 1983):

- „Coercive isomorphisms“ resultieren aus politischem Druck und der interdependenten Abhängigkeit zwischen Organisationen, insbesondere weil Unternehmen ihre Entscheidungen gegenüber internen (z.B. den Beschäftigten) und externen (z.B. Investoren, Zulieferern oder Regierungsbehörden) Stakeholdern legitimieren müssen. Bei Nichteinhaltung rechtlicher oder gar informaler Vereinbarungen drohen Sanktionen und Ansehensverlust.

- „Mimetic processes“ werden als der Hauptgrund angesehen, warum es weltweit zur Verbreitung von Managementmoden, „best practices“ und der Einführung immer ähnlicherer Organisationsstrukturen und -prozessen kommt. Es wird angenommen, dass immer komplexer werdende Technologien, Zielambiguität und Umweltunsicherheiten Manager in Organisationen dazu ermuntern, erfolgreiche 
Unternehmen zu kopieren, um selbst erfolgreich zu erscheinen und die Legitimität ihres Handelns zu erhöhen.

- „NNormative pressures“ werden vor allem darin gesehen, dass moderne kapitalistische Staaten immer professionalisierter werden, was mit einem Bedeutungszuwachs von bestimmten Berufsgruppen und -verbänden einhergeht, z.B. von Accountants im anglo-sächsischen Kontext. Vor allem zwei Aspekte der Professionalisierung werden hervorgehoben: Zum ersten die zunehmende Bedeutung formaler Bildungsabschlüsse, insbesondere von Abitur und Hochschulabschlüssen, um überhaupt bestimmte Karrierechancen in Unternehmen zu haben bzw. auf bestimmte Managementpositionen zu gelangen. Zum anderen sind professionelle Netzwerke, wie Berufs-, Bildungs- oder Industrieverbände, über Landesgrenzen hinweg immer mächtiger und einflussreicher.

Anders als von Hofstede und den europäischen Institutionalisten angenommen, besteht das Resultat solcher isomorphen Prozesse eben nicht in kultureller und institutioneller Vielfalt nationaler und lokaler Rationalitäten, sondern in zunehmender Homogenität. In bezug auf die Thematik dieses Beitrages und ausgehend von den Grundannahmen des amerikanischen Institutionalismus stellt sich nun erstens die Frage, ob und inwieweit international operierende Unternehmen noch von nationalen Institutionen beeinflusst werden, d.h. einerseits von der institutionellen Einbettung in ihrem Herkunftsland und andererseits durch die verschiedenartigen nationalen Institutionengefüge in den Ländern, in denen sie Niederlassungen haben. Zweitens wäre zu fragen, inwieweit die drei Formen des Isomorphismus die Herausbildung kulturell homogener transnationaler Managementrollen und Organisationsformen in MNU befördern. Auf beide Fragen wird im folgenden Abschnitt näher eingegangen.

\section{Lokale und globale Isomorphismen, „National Business Systems“ und das multinationale Unternehmen}

\subsection{Institutionelle Relativierung des evolutionären Paradigmas}

Es gibt vielfältige Ansätze, insbesondere und besonders intensiv im USamerikanischen internationalen Management Diskurs, welche die evolutionäre Theorie mit Kernideen des amerikanischen Institutionalismus anzureichern versuchen (Ghoshal/Bartlett, 1993; Nohria/Ghoshal, 1997). Die Kernthese der 
netzwerkorientierten Ansätze ist, dass neben der Aufgabenumwelt die institutionelle Umwelt eine zunehmende Rolle spielt. Im Mittelpunkt steht also die Frage, wie weniger hierarchische, eher dezentralisierte und horizontal koordinierte transnationale Unternehmen funktionieren. Obwohl in diesem Zusammenhang zwar auch darauf verwiesen wird, dass insbesondere lokale Isomorphismen und Legitimierungsprobleme die MNU zu lokalen Anpassungen in den Niederlassungen ihrer Gastländer zwingen, wird verstärkt die Wirkung globaler Isomorphismen in transnationalen Unternehmen hervorgehoben. Diese werden zunehmend als Weiterentwicklung des ursprünglichen Ansatzes interpretiert, also als „differenzierte Netzwerke“, welche zu Konvergenz von Strukturen und Kommunikationsprozessen tendieren. In bezug auf den Ansatz von DiMaggio und Powell (1983) und Levitts (1983) These von der Welt als „global village“ wird angenommen, dass mimetische und normative isomorphe Mechanismen in differenzierten Netzwerken stärker zur Geltung kommen.

Die beschriebenen Versuche, vor allem von Hauptvertretern dieser Forschungsrichtung, einige der Grundaussagen des amerikanischen Institutionalismus zu integrieren, haben jedoch die Kernthesen des evolutionären Modells (insbesondere den normative Bias) nicht grundsätzlich in Frage gestellt. Danach nähern sich international operierende Firmen mit steigendem Grad der Globalisierung ihrer Geschäftstätigkeiten zunehmend einem ,transnationalen` Modell an. Dieses erscheint nicht nur als die beste Lösung für das Dilemma von MNU, gleichzeitig global zu integrieren und dennoch lokal angepasst zu bleiben, sondern auch als der (neue) „one best way“, um weltweites Lernen und Innovationen über nationale Ländergrenzen hinweg zu befördern (Bartlett/Ghoshal, 1997; Macharzina/Oesterle/Brodel, 2001). Interessanterweise haben sich die Integrationsversuche der evolutionären Theorien der MNU mit institutionalistischen Theorien bisher ausschließlich auf den amerikanischen Institutionalismus beschränkt. Eine Rezeption des europäischen Institutionalismus in der US-amerikanischen Debatte zu MNU hat bisher faktisch nicht stattgefunden. Darauf wird noch zurückzukommen sein.

Aus institutioneller Perspektive ergeben sich neben der eingangs diskutierten generellen Kritik an den Konvergenzannahmen universeller und kontingenztheoretischer Theorien insbesondere folgende Probleme, welche die Erklärungsmacht des über 30 Jahre in der internationalen Managementforschung dominanten evolutionären Modells erheblich dezimieren: 
- D Die evolutionäre „one best best way“ Logik versperrt den Weg für eine differenziertere Betrachtung der MNU: insbesondere darauf, warum es jenseits technologischer und ökonomischer Zwänge durchaus Sinn für international operierende Firmen macht, nicht oder nur in eine geringe Anzahl von Ländern zu expandieren (Westney/Zaheer, 2001) bzw. in einem der „früh-evolutionären“ Stadien zu verharren (Geppert/Matten/Williams, 2003).

- $\quad$ Auch wenn US-amerikanische Firmen am weitaus häufigsten unter den am meisten globalisierten Unternehmungen zu finden sind, gibt es eine wachsende Zahl von international erfolgreich operierenden Firmen aus unterschiedlichen Herkunftsländern, die nicht unbedingt dem evolutionären Modell vom multinationalen zum transnationalen Stadium folgen (Westney/Zaheer, 2001). ${ }^{4}$

- $\quad$ Der starke „unilateral, monotonic bias towards international expansion“ (Westney/Zaheer, 2001: 369) evolutionärer Ansätze der MNU verhindert auch eine differenziertere Betrachtung institutioneller Vielfalt der Einflüsse und Rationalitäten z.B. durch das NBS des Gastlandes der Niederlassungen (Geppert/Williams/Matten, 2003).

Auf die letzten beiden Aspekte wollen wir nun im weiteren detaillierter eingehen. Wie zu zeigen sein wird, werden sowohl „home country“ als auch „host country effects“ in beiden institutionalistischen Ansätzen diskutiert. Der große Unterschied jedoch ist, dass der europäische Institutionalismus historisch durch international vergleichende Studien gewachsen ist und auf eher qualitativen Forschungsmethoden basiert. Der amerikanische Institutionalismus bezieht sich jedoch weitgehend auf quantitative Analysen von Populationen von Organisationen (organizational forms) und beschränkt sich auf einen Nationalstaat, meistens die USA (Rosenzweig/Singh, 1991; Tempel/Walgenbach, 2003). Wie wir im weiteren sehen werden, hatte diese Herangehensweise der Theoriekonstruktion eine vergleichsweise einschränkende Wirkung auf die Erforschung der Organisation international operierender Firmen und damit auch auf das Verständnis der Rolle der institutionellen Bedingungen des Herkunftslandes und der Gastländer. ${ }^{5}$

\subsection{Rolle des Heimatlandes von MNU (Country of origin effects)}


Wie wir gesehen haben wird der Rolle des Heimatlandes, insbesondere von evolutionären Theorien in den höheren (internationalen und transnationalen) Stadien der Entwicklung der MNU eine zunehmend verschwindende Bedeutung zugewiesen. Bartlett und Ghoshal verweisen zwar auf die bleibende Bedeutung des ,administrative heritage“ im MNU, konzentrieren sich aber in ihrer Analyse mehr auf die Frage, warum und wie Manager in global agierenden Firmen neben transnationalen Strukturen Unternehmensprozesse und eine globale Kultur (bzw. "global mentality") entwickeln müssen (Bartlett/Ghoshal, 1989: 35-55). Diese Diskussion wird von amerikanischen Institutionalisten dahingehend aufgegriffen, dass Strategien von MNU auf die kulturelle bzw. die institutionelle Distanz des Herkunftslandes zurückzuführen sind. Das erste Konstrukt geht dabei mit Hofstede davon aus, dass insbesondere die Toleranz für Unsicherheit zwischen Nationalstaaten erheblich differiert und damit die Art und Weise, ob und wie die Mutterunternehmen ihre Niederlassungen integrieren bzw. kontrollieren. Es wird angenommen, dass vor allem formale Kontroll- und Koordinationsformen positiv mit kultureller Distanz korrelieren (Rosenzweig/Singh, 1991). Neuere Studien haben diese Idee verfeinert und verweisen darauf, dass nicht kulturelle Unterschiede zwischen Herkunftsland und Gastland der MNU darüber entscheiden welche strategischen Entscheidungen getroffen werden. Es ist vielmehr auch gewichtig, ob die Strategien in der Niederlassung als legitim anerkannt werden. Die Frage der institutionellen Distanz wird somit an den institutionellen Unterschieden (insbesondere im Rechtssystem) des Gastlandes zum Herkunftsland der MNU festgemacht bzw. ganz im Stile evolutionärer Theorien mit strukturellen Kontingenzen der MNU in Bezug auf ihre Aufgabenumwelt (wie z.B. Industriestruktur, Organisationsstruktur, Eigentumsverhältnisse etc.) erklärt (Xu/Shenkar, 2002). Dabei werden zwei Arten institutioneller Umwelten unterschieden: 1. die institutionelle Umwelt des Gastlandes und 2. das strukturelle Design des MNU, welches als „,institutional field“ interpretiert wird (Rosenzweig/Singh, 1991; Westney, 1993). Damit wird eine neue Antwort auf das alte Dilemma international operierender Firmen gegeben. Die richtige Mischung zwischen globaler Integration und lokaler Anpassung kann eben nicht allein mit ökonomischen und technischen Zwängen erklärt werden. Sie ist darüber hinaus davon abhängig, wie sich Manager in MNU die Legitimität ihrer Handlungen intern, also gegenüber anderen Abteilungen und Niederlassungen der MNU und extern, also gegenüber den relevanten Stakeholdern im Gastland, herstellen. Im Gegensatz zum europäischen Institutionalismus wird das Institutionengefüge des Herkunftslandes als 
gegeben angesehen. Diskutiert wird lediglich die institutionelle Distanz des strategischen Ansatzes der Muttergesellschaft im Verhältnis zum Gastland. Hierbei werden sowohl regulative, normative als auch kognitive Elemente institutioneller Einflüsse berücksichtigt. In diesem Sinne wird angenommen, dass die MNU (Xu/Shenkar, 2002):

- $\quad$ in „global industries“ eher in ähnlichen institutionellen Umwelten, mit ähnlichen normativen und kognitiven institutionellen Mechanismen, investieren. MNU in „multidomestic industries“ arrangieren sich dagegen in institutionell verschiedenen Umwelten.

- $\quad$ mit größerer institutioneller und struktureller Vielfalt dazu tendieren, toleranter gegenüber verschiedenen Normen und Regeln in ihren ausländischen Niederlassungen zu sein. Daher existiert auch eher eine Bereitschaft, dort zu investieren. Homogene MNU operieren eher in ihren Heimatländern ähnlichen Kontexten.

- $\quad$ dort, wo die normative und kognitive institutionelle Distanz zum Gastland groß ist, eher zu sogenannten „greenfield investments“ neigen. Akquisitionen finden statt, wo diese Distanz eher klein ist.

- $\quad$ eher dazu bereit sind, eine Mehrheit bzw. die volle Eigentümerschaft in einem Joint ventures zu übernehmen, wenn die regulative und normative institutionelle Distanz gering ist.

Auch die MNU selbst wird in dieser Familie von institutionellen Ansätzen als institutionelles Feld thematisiert und damit, abhängig von bestimmten Konstellationen kultureller oder institutioneller Distanz, als mehr oder weniger global ausgerichtet gesehen. MNU gehen i.d.R. eben über einen Nationalstaat hinaus und sind damit eher transnationaler Natur. Diese Ausrichtung des amerikanischen Institutionalismus hat jedoch auch deutliche Grenzen, wie vor allem Ferner und Qunitanilla in bezug auf den Einfluss des Herkunftslandes in MNU deutlich machen. Insbesondere wird die ahistorische und die statische Beschreibung von Nationalstaaten kritisiert, die sich, wie schon angemerkt wurde, dominant auf die nationalen Unterschiede im Gastland konzentriert. Sie kommen daher zu folgendem Schluß: ,it seems ironic that writers using an ,institutionalist perpective to analyse MNCs should not focus more on institutional differences between countries“ (Ferner/Quintanilla, 1998: 714). 
Im Unterschied zum amerikanischen Institutionalismus ist dieses Thema eine zentrale und traditionell wichtige Fragestellung im europäischen Institutionalismus. Dafür gibt es vielfältige Gründe. Ein wichtiger Aspekt ist dabei die Analyseebene, die eben beim europäischen Institutionalismus explizit auf den Nationalstaat und das Institutionengefüge des NBS bezogen ist und nicht auf „organizational fields“, wie bestimmte industrielle Sektoren. Hier liegt das Verdienst des europäischen Institutionalismus. Ausgehend von der Grundannahme, dass es keinen „one best way“ der Organisation ökonomischer Aktivitäten gibt, wird die Existenz eines einheitlichen Funktionsmechanismus für kapitalistische Institutionen, wie er im amerikanischen Institutionalismus durchscheint, bestritten. Eine Kernfrage ist daher, inwieweit international operierende Firmen von den Wettbewerbsvorteilen des NBS des Heimatlandes profitieren. Diese Frage bekommt vor dem Hintergrund der Dominanz des evolutionären Models in der internationalen Managementforschung, wobei die „transnationale Lösung“ als „best practice“ oder der neue „one best way“ propagiert wird, besondere Brisanz. Insofern kann das Aufzeigen von „country-of-origin effects“ auch als Versuch begriffen werden, nicht nur die institutionellen Einflüsse des Herkunftslandes aufzuzeigen. Vielmehr sind MNU niemals völlig integriert oder kulturell homogen, sondern existieren eher als ein Geflecht verschiedener „kontextueller Rationalitäten“ (Morgan, 2001). Die Diskussion über den verbleibenden Einfluss nationaler Rationalitäten verweist auch auf verstärkte Spannungen und Konflikte. Diese werden insbesondere dann akut, wenn Firmen internationalisiert werden. Ein Prozess, den Ferner und Qunitanilla mit „AngloSaxonization“ (Ferner/Quintanilla, 1998) bezeichnet haben. Studien, die den verbleibenden Einfluss des NBS des Herkunftslandes in MNU trotz Tendenzen der „Anglo-Saxonization“ betonen, beschäftigen sich vor allem mit den folgenden Problemkomplexen: internationale Akquisitionen (Child/Faulkner/Pitkethly, 2001), HRM Praktiken (Ferner, 1997; Edwards/Ferner, 2001; Gunnigle et al., 2002), Kontrolle und Koordination (Ferner, 2000; Becker-Ritterspach/Lange/Lohr, 2002; Harzing/Sorge, 2003) und der Wandel von Arbeitssystemen (Sorge, 1995; Geppert/Matten/Williams, 2003; Geppert/Williams/Matten, 2003).

Wir wollen uns hier exemplarisch auf den letzten Aspekt konzentrieren und werden dabei hauptsächlich auf die aktuellen Ergebnisse der eingangs erwähnten international vergleichenden Studie über Veränderung von Arbeitssystemen in MNU zurückgreifen. In der komparativen Studie wurde untersucht, wie ein deutscher, finnischer und US-amerikanischer MNU in derselben industriellen Branche ihre 
Arbeitssysteme weltweit reorganisieren. D.h., es geht konkret um die Analyse von Veränderungen in Struktur und Entscheidungsprozessen sowie Technik- und Personaleinsatz. Besonderes Augenwerk wurde dabei der Beziehung der Muttergesellschaften zu ihren Niederlassungen geschenkt.

Es fanden sich zwar durchaus einige globalen Tendenzen in allen drei MNU, wie z.B. die zunehmende Bedeutung finanzieller Aspekte im Managementdiskurs (Geppert, 2002), die zu einer verstärkten Orientierung der MNU auf den im Vergleich zur Fertigung ertragsstärkeren Bereich Service \& Instandhaltung führten. Hier gibt es durchaus Bezugspunkte zu normativen und kognitiven Elementen des amerikanischen Institutionalismus. Eine Haupterkenntnis der internationalen Vergleichsstudie ist aber der Nachweis des bleibenden Einflusses des Herkunftslandes auf die globalen und lokalen Strategien zur Restrukturierung der Arbeitssysteme. Im Falle deutscher MNU besteht auch weiterhin eine Tendenz zu Produktionssystemen, die Sorge und Streek (1988) als diversifizierter Qualitätsproduktion (im folgenden DQP) definiert haben. Einhergehend mit einer Ansiedlung der Produktpalette im oberen Marktsegment sind die wertschöpfungsintensiven Arbeitsvorgänge in solchen Firmen eng mit Kundenorientierung und F\&E verzahnt. Das Arbeitssystem spiegelt wichtige Elemente des deutschen NBS wider, wie z.B. langfristige strategische Orientierung, hoher Stellenwert beruflicher Bildung, formale Aus- und Weiterbildung und verbriefte Rechte von Arbeitnehmern. Diese Elemente ermöglichen die kontinuierliche Verbesserung sowohl von Arbeitsprozessen als auch von Produkten und Produktprogrammen. Im Gegensatz dazu setzte die US-amerikanische MNU auf globale Standardisierung von Strukturen, Prozessen und Produkten sowie die weltweite Rationalisierung der Produktion. Diese Befunde decken sich mit den Ergebnissen einer andern komparativen Studie. Darin kommen die Autoren zu dem Ergebnis, dass US Firmen ihre HRM Praktiken stärker standardisieren und weniger bereit sind, diese an lokale Bedingungen anzupassen (Gunnigle et al., 2002). Diese Strategie spiegelt natürlich auch die eingangs beschriebenen Tendenzen der AngloSaxonization wider, allerdings auf sehr US-amerikanische Weise. Da der amerikanische Konzern als einziger der untersuchten Unternehmen an der New Yorker Börse gelistet ist, schlägt die kurzfristige Finanzorientierung stärker durch. Daneben sind auch Zielvereinbarungen im Management, Budgetierung und Entlohnungssysteme typisch amerikanisch auf kurzfristige Gewinnmaximierung ausgerichtet. 
Die Strategie der finnischen MNU hat viele Ähnlichkeiten mit der USamerikanischen. Die Strategien beider MNU sind im Sinne von Porter auf Kostenführerschaft ausgerichtet, wobei die deutsche MNU in ihrem Produktionsmodell klar eine auf Qualität fokussierte Differenzierungsstrategie verfolgt (Porter, 1980). Trotz alledem kann die „Anglo-Saxonization“ der finnischen MNU aus den tiefgreifenden Veränderungen im finnischen NBS erklärt werden (Lilja/Räsänen/Tainio, 1992). Wie Tainio und Koautoren zeigen, hat die verstärkte Öffnung des Landes für internationale Investoren und damit auch die Bedeutung von Shareholder Value zu einer Amerikanisierung vieler finnischer MNCs geführt (Tainio/Huolman/Pulkkinen, 2001). Allerdings zeigt sich der bleibende Einfluss des NBS an der starken Technologieorientierung der MNU (sie ist Technologieführer im Fahrstuhlbereich) und der engen Einbindung finnischer Manager in die interorganisationalen Netzwerke des Herkunftslandes. Das deckt sich mit der Einschätzung von Lilja und Koautoren, dass technische Exzellenz und Innovation obere Priorität in der finnischen Industriepolitik hatten und weiterhin haben werden. Insbesondere das finnische Beispiel stellt einen signifikanten Unterschied in der Theoretisierung des Einflusses von Institutionen und MNU dar. Der europäische Institutionalismus ist in der Lage zu zeigen, wie Veränderungen in der institutionellen Umwelt, im NBS, auf die Entstehung interner normativer aber auch politischer Prozesse Einfluss nehmen. Im Falle des amerikanischen Institutionalismus dagegen konzentriert sich die Dynamik auf institutionelle Veränderungen im institutionellen Feld des MNU. Eine externe institutionelle Veränderung des Herkunftslandes kommt entweder nicht vor oder ist statisch auf das Auftauchen der drei Isomorphismen festgelegt.

\subsection{Macht und Autonomie in Gastland-Niederlassungen (host country subsidiaries)}

Das Interesse an der Rolle der Niederlassungen in MNU ist vor allem durch die Kritik an den frühen, eher hierarchisch orientierten, Herangehensweisen evolutionärer Ansätze entstanden. Sowohl die weitere Entwicklung dieses Ansatzes durch Hedlunds “Heterarchy” Konstrukt (Hedlund, 1986), als auch die Diskussion von MNU als differenzierte Netzwerke von Nohria und Goshal (1997) haben dazu geführt, dass die vormals eher als passiv definierte Rolle von Niederlassungen hinterfragt - und 
insbesondere durch die Forschungen von Birkinshaw und Koautoren (Birkinshaw/Fry, 1998; Birkinshaw/Hood, 2001) - neu definiert wurde. In verschiedenen Beiträgen wird hier zunächst an die Ideen der evolutionären Theorie angeknüpft. Allerdings geht die Diskussion um „subsidiary evolution“ darüber hinaus (Birkinshaw/Hood, 1998). Es wird gezeigt, dass Niederlassungen in MNU nicht nur Autonomie brauchen, um innovativ zu sein oder gar „entrepreneurship“ zu entwickeln. Darauf aufbauend wird dann weiter diskutiert, wie Niederlassungen diese durch „charter enhancement“ erringen können. So wird gezeigt, dass die Autonomie von Niederlassungen wächst und damit auch ihre Innovationsfähigkeit sowie unternehmerisches Handeln im Management. Voraussetzung dafür ist, dass die Niederlassung Kernkompetenzen hat, die sie gegenüber anderen Unternehmenseinheiten wettbewerbsfähiger macht. Ausserdem ist das Vorhandensein eines „strong track record“ notwendig. Des weiteren wurde beobachtet, dass die Entscheidungsprozesse in der gesamten MNU dezentraler organisiert sind, die strategische Orientierung der Muttergesellschaft nicht ethno-zentristisch ist und die Kommunikationsbeziehungen zwischen Mutter- und Tochtergesellschaft eine hohe Qualität haben (Birkinshaw/Hood, 1998). Soweit zu den internen Faktoren, die die Autonomie und damit auch die Machtposition der Niederlassung in der MNU erhöhen. Darüber hinaus werden, ganz im Stile der evolutionären Theorie, aber auch die strukturellen und ökonomischen Bedingungen des Gastlandes genannt, die sowohl positiven als auch negativen Einfluss auf die Autonomie der Niederlassung haben können. Genannt werden hier:

- $\quad$ die Dynamik des Markes und die zusätzlich entstehenden Kosten für die MNU im Gastland

- $\quad$ die strategische Wichtigkeit des Gastlandes für die MNU

- $\quad$ die Bereitschaft des Gastlandes, die Aktivitäten der MNU durch Fördermittel zu unterstützen.

Alles in allem können wir zusammenfassen, dass die Idee der „subsidiary evolution“ eine Weiterentwicklung des evolutionären Ansatzes darstellt. Dieser setzt sich stärker mit der Rolle der Niederlassungen auseinander. Ein Aspekt, der in der MainstreamDiskussion vernachlässigt wurde. Inwieweit die institutionelle Umwelt die Autonomie und Macht der Niederlassungen beeinflusst, wird nur am Rande angerissen (z.B. mit Verweis auf die regulative Funktion des Staates im Gastland), jedoch nicht systematisch untersucht. 
Wie schon im vorigen Abschnitt angemerkt, wird der Rolle der institutionellen Umwelt des Gastlandes im amerikanischen Institutionalismus eine größere Bedeutung beigemessen als dem Herkunftsland. Die Grundnahme ist, dass die Niederlassungen sich vor allem aufgrund regulativer und normativer Zwänge an die institutionelle Umwelt des Gastlandes anpassen müssen. Betont wird auf der einen Seite, dass sich Managementkonzepte, die in einem ganz bestimmten nationalen Kontext entwickelt wurden, über interne isomorphistische Prozesse im ganzen Unternehmen ausbreiten und somit legitime „best practices“ werden. Allerdings wird auf der anderen Seite auch herausgestellt, dass die MNU Einfluss auf die Institutionenbildung im Gastland nehmen kann und auch nimmt. So wird etwa das Beispiel der Hotel-Luxuskette Marriott angeführt, die nach ihrer Eröffnung eines Hotels in Hong-Kong Einfluss auf die Regulierung der Wochenarbeitszeit nahm (Rosenzweig/Singh, 1991: 353-354 ). ${ }^{6}$ Letztendlich wird damit auch der Rückgang des Einflusses der nationalen Institutionen erklärt. Oder anders formuliert: Die wachsende Macht der MNU auf nationale Gesetzgebung und die Finanzierung vormals öffentlicher, d.h. staatlich finanzierter Dienstleistungen durch MNU, wird als Indiz dafür angesehen, dass spezifische national geprägte institutionelle Unterschiede immer mehr verschwinden. Eine Schlussfolgerung daraus ist, dass im Gegensatz zum europäischen Institutionalismus von der Entstehung eines „global inter-corporate isomorphism“ ausgegangen wird (Ferner/Quintanilla, 1998). Die Annahme ist dabei, es finden sich MNU in nationalen Umwelten wieder, die einander ähneln (Rosenzweig/Singh, 1991). Solche isomorphistischen Prozesse werden auch für die Entstehung transnationaler Räume verantwortlich gemacht. Darauf werden wir im nächsten Abschnitt näher eingehen.

Studien in der Tradition des europäischen Institutionalismus lassen jedoch Zweifel darüber aufkommen, der Einfluss der Gastländer würde gänzlich schwinden. Interessant sind hier insbesondere die Ergebnisse einer vergleichenden Studie zur Einführung von Gruppenarbeit in deutschen und französischen Niederlassungen in der Automobilindustrie (Woywode, 2002). So stellt Woywode zwar fest, dass sich durchaus isomophistische Tendenzen in den Niederlassungen finden lassen, welche sich global durch den gesamten industriellen Sektor ziehen. Genannt werden kann hier z.B. das Gruppenarbeitskonzept, welches ursprünglich in japanischen Automobilfertigungsstätten zur Anwendung kam. Dieses ist nun in der gesamten Branche über Ländergrenzen hinweg anzutreffen. Allerdings weist Woywode auch nach, dass die lokalen Anpassungen eine starke Einfärbung durch das NBS in einem 
Sektor erfahren, der gerade von vielen als Musterbeispiel für das Vorhandensein globaler Industrien (Rosenzweig/Singh, 1991: 349) bzw. als ein „continuous globalizing“ Sektor (Whitley/Kristensen, 1997) angesehen wird. In diesem Sinne wurde festgestellt, dass insbesondere die Unterschiede im Bildungssystem und in den industriellen Beziehungen für einen höheren Grad an multiplen Qualifikationen bei den Gruppenmitgliedern, mehr Zusammenarbeit in der Gruppe, mehr Bereitschaft zu „job rotation“ und damit auch eine höhere Gruppenautonomie in den deutschen Niederlassungen verantwortlich waren.

Diese Befunde decken sich weitgehend mit den Erkenntnissen aus dem Vergleich britischer und deutscher Niederlassungen von MNU in der Fahrstuhl- und Rolltreppenfertigung. Zwar lässt sich in den deutschen und britischen Niederlassungen aller drei MNU in der Tat eine zunehmende Finanzorientierung und ein verstärkter Trend zum Ausbau der Servicefunktionen im Management feststellen (Geppert/Matten/Williams, 2003). Gleichzeitig jedoch zeigte ein Vergleich der Reorganisation der Arbeitssysteme in beiden Ländern starke Unterschiede sowohl in der Veränderung von Strukturen und Entscheidungsprozessen sowie dem Einsatz von Fertigungstechnologien und Personal. Damit wird trotz Globalisierungstendenzen die bleibende Bedeutung unterschiedlicher NBS unterstrichen. In den deutschen Niederlassungen hatten die Arbeitssysteme aufgrund der engeren Interdependenz von arbeitsmarktpolitischen, bildungspolitischen und gewerkschaftlichen Institutionen eine starke Tendenz zu DQP. In Großbritannien dagegen ist das NBS weniger eng mit den Arbeitsystemen verzahnt. D.h. konkret, dass die industriellen Beziehungen eher schwach und die arbeitspolitischen Maßnahmen eher unterentwickelt sind. Die berufliche Bildung und Weiterbildung, insbesondere von Facharbeitern, Technikern und Ingenieuren, hat hier einen geringen Stellenwert. Die Beschäftigten in den Fertigungsstätten waren daher eher angelernt oder ungelernt und weniger in Managementprozesse einbezogen. Darüber hinaus verfügten Personen in strategischen Entscheidungspositionen zumeist über einen Managementabschluss. Im Gegensatz dazu werden in deutschen Niederlassungen diese Managementfunktionen mehrheitlich von Ingenieuren wahrgenommen. Ein massiver Abbau von „bad performing“ Fertigungskapazitäten und eine Reorientierung der Funktionen des Arbeitssystems auf das finanziell weitaus lukrativere Geschäft in Verkauf und Service erschien somit vielen der befragten Managern als die beste Lösung

(Geppert/Williams/Matten, 2003). 
Im Lichte von Birkinshaws Entrepreneurship Konzept lässt sich abschließend resümieren, dass die deutschen Niederlassungen in beiden Studien größere „,harter responsibilties“ haben. Somit haben die lokalen Manager dort auch größere Entscheidungsmacht und Autonomie. Des weiteren bleibt festzuhalten, dass von der Vogelperspektive (bzw. einer abstrakteren Ebene), die MNU in den beiden zuletzt diskutierten Studien durchaus globale isomorphistische Tendenzen aufweisen. Hier kommen dann auch die Stärken des amerikanischen Institutionalismus zum Tragen. Verlässt man jedoch diese Abstraktionsebene und versucht, die interaktiv erzeugten und sozial konstruierten lokalen Managementrationalitäten zu verstehen, entwickelt sich ein besseres Verständnis für die „feinen Unterschiede“. Erst die Wahl eines solchen methodisch interpretativen Ansatzes macht es möglich zu erkennen, warum und auf welche Art und Weise der Einfluss von nationalen Institutionen auch weiterhin von Bedeutung sein wird. In diesem Sinne haben Geppert und Koautoren darauf verwiesen, dass, im Gegensatz zu den Annahmen der evolutionären Theorie, nationale Effekte in transnationalen Unternehmen eben nicht zunehmend verblassen. Vielmehr kommen die nationalen Unterschiede der Niederlassungen in der MNU eher noch stärker zum Tragen (Geppert/Matten/Williams, 2003). Darüber hinaus wird argumentiert, dass MNU diese Differenzen strategisch sogar ganz bewusst in Kauf nehmen bzw. ausnutzen. Dies ist z.B. der Fall, wenn sie ihre globalen Fertigungskapazitäten rationalisieren. Der Vergleich von MNU in der Fahrstuhl- und Rolltreppenfertigung beispielsweise zeigte die bewusste Nutzung von kompetitiven Vorteilen und Kapazitäten von Arbeitsystemen im deutschen NBS. Im Vergleich zu den britischen Unternehmen hatten die Deutschen eher strategische Funktionen in der MNU inne und waren gleichzeitig für die innovativen F\&E Aktivitäten verantwortlich (Matten/Geppert, 2002, 2004).

\subsection{MNU als „transnationale soziale Räume“: Grenzen institutioneller Ansätze und Herausforderungen für zukünftige Forschung}

Ein wichtiger Zweig in der aktuellen sozialwissenschaftlichen Debatte (Pries, 2001; Morgan, 2001b) zur Rolle von MNU ist die Frage nach der Entstehung von „transnationalen sozialen Räumen“ (Morgan, 2001). Die Kernfrage dieser jüngeren Forschungsrichtung im Internationalen Management zielt auf die Entstehung und Veränderung von makro-institutionalistischen Strukturen durch soziale Praktiken 
sozialer Akteure ab. Die Institutionalisierung von „transnationalen sozialen Räumen“ wird hier als ein sozio-politischer Prozess konzeptualisiert, der verschiedene Formen sozialen Handelns beinhaltet (wie z.B. Zwang, Widerstand und Verhandlung, siehe Geppert/Clark, 2003). In diesem Sinne richtet sich die Erforschung „transnationaler soziale Räume“ auf die Frage aus, wie die Grenzen von „transnational communities“ in und außerhalb von MNU strukturiert sind, gemanagt werden und wie diese Einheiten sich (re-)definieren und in Verhandlungen (z.B. zwischen externen Stakeholdern wie Gewerkschaften oder Banken oder zwischen verschiedenen Einheiten innerhalb der MNU selbst) ausgehandelt werden. Transnationale Räume entstehen im Wechselspiel von kulturellen und institutionellen Kontexten, durch ausgehandelte formale und informale Regeln, durch Zugang oder den Besitz von bestimmten Fähigkeiten oder Kapazitäten und Ressourcen (z.B. in den Niederlassungen), die durch sozio-politische Prozesse zusammen gehalten werden oder auch wieder auseinander brechen können. Für die Theorie der MNU und die Erforschung „transnationaler sozialer Räume“ entstehen damit eine Reihe von Fragestellungen.

Wiewohl die Forschung zu transnationalen sozialen Räumen beiden hier diskutierten Institutionalismen konzeptionell eine Heimat bietet, wird die Frage nach globaler Konvergenz bzw. divergenten national-spezifischen Unterschieden jüngst nicht mehr allein durch Rückgriff auf abstrakte isomorphistische makro-institutionelle Mechanismen oder die Makro-Institutionen des Nationalstaates zu beantworten versucht. Mikro-institutionelle Vertreter dieser Richtung kritisieren den eher deterministischen Bias institutionalistischer Ansätze und verweisen darauf, dass Institutionen nicht nur einschränkende, sondern auch ermöglichende Wirkung auf Strukturen und Handlungen in Unternehmen haben. Sie schlagen daher vor, transnationale soziale Räume nicht nur als institutionalisierte Arenen, die sich nicht grundsätzlich verändern, sondern auch als institutionalisierende Arenen, in denen selbst ständig neue Institutionalisierungsprozesse stattfinden, zu definieren (Djelic/Quack, 2002). D.h., zukünftige Forschung sollte nicht nur darauf abstellen, wie bestimmte nationale Institutionen MNU beeinflussen, sondern auch, wie signifikante Veränderungen nationaler Institutionen die Strategien von MNU in diesem NBS beeinflussen. Dieser Aspekt wurde bereits kurz in den oben erwähnten Ergebnissen des Forschungsprojektes von Geppert und Koautoren (Geppert/Williams/Matten, 2003) im Falle der finnischen MNU aufgegriffen und diskutiert. Aus deutscher Perspektive stellt die Diskussion insbesondere auf die Frage 
ab, inwieweit „der Standort Deutschland“ - oder institutionalistisch gesprochen: das deutsche NBS - für MNU mit dem Heimatland Deutschland noch in der Lage ist, Rahmenbedingungen erfolgreicher unternehmerischer Aktivität zu bieten (Bergedorfer Gesprächskreis, 2000).

Eine weitere Fragestellung in diesem Kontext zielt darauf ab, in wieweit MNU direkt und aktiv Einfluss auf die Entstehung und Veränderung nationaler und transnationaler Institutionen nehmen. Eine Frage, die - wie wir oben gezeigt haben - von Vertretern des amerikanischen Institutionalismus diskutiert wird. Hier besteht allerdings noch erheblicher Forschungsbedarf, da weitaus differenzierter aufzuzeigen wäre, auf welche Art und Weise machtvolle soziale Akteure (d.h. einflussreiche transnational agierende Eliten und kollektive Akteure) die Wirkungsweise der drei Isomorphismen strategisch vorantreiben. In diesem Sinne ist die Entstehung „transnationaler sozialer Räume“ in und durch MNU eher eine Frage die sich auf dem mikro-institutionellen Level abspielt, und ist damit zutiefst politisch. Als solcher ist dieser Aspekt in der evolutionären Theorie der MNU und den institutionalistischen Ansätzen bisher eher vernachlässigt worden. Die Idee des „transnationalen sozialen Raumes“ in und durch MNU geht davon aus, dass diese durch verschiedene Strategien machtvoller Akteure entstehen (Morgan, 2001; Geppert/Clark, 2003). Der Focus liegt also nicht nur darauf, ob sie mehr oder weniger institutionalisiert sind (Scott, 1998) bzw. eine hohe oder geringe institutionelle Dichte aufweisen (Whitley, 1997).

Es bleibt allerdings abzuwarten, inwieweit sich die hier behandelten institutionalistischen Ansätze in Zukunft diesen neuen Herausforderungen stellen können und dazu beitragen werden, die Dynamik sozialen Handelns im Prozess der Institutionalisierung „transnationaler sozialer Räume“ besser einzufangen. Es kann jedoch vermutet werden, dass beide Ansätze von unterschiedlichen konzeptionellen Prämissen und methodischen Blickwinkeln an diese Probleme herangehen und daher auch weiterhin eher zu unterschiedlichen empirischen Befunden zu Organisation und Management in und von MNU kommen werden.

\section{Abbildung 2 etwa hier}

\section{Zusammenfassung und Ausblick}


Abbildung 2 bietet einen Überblick über die wesentlichen Aussagen institutionalistischer Theorieansätze zu den in diesem Beitrag ausgewählten Forschungsfragen im Kontext der MNU. Darüber hinaus deutet die unterste Zeile ansatzweise an, in welcher Beziehung die Arbeit institutionalistischer Forscher zu den tradierten kontingenztheoretischen Ansätzen zu sehen ist. Allerdings folgen wir im Sinne einer zusammenführenden Beurteilung der Sicht von Geppert und Koautoren. Deren Argumentation (an anderer Stelle) ist, dass diese Ansätze nicht in einer wechselseitige Ausschließlichkeit zu sehen sind, sondern vielmehr als Ergänzung des tradierten theoretischen Instrumentariums vorgeschlagen werden (Geppert/Matten/Williams, 2003).

Für die weitere institutionalistische Managementforschung sehen wir zukünftiges Forschungspotential vor allem auf drei Ebenen. Zunächst besteht auf Unternehmensebene weiter großer Bedarf, das zuletzt angedeutete Spannungsfeld der MNU als einem Akteur zwischen globalen Isomorphismen auf der einen Seite und kontextuellen Rationalitäten und Politiken verschiedener lokaler Akteure mit unterschiedlichen nationalen Hintergründen auf der anderen Seite weiter und differenzierter auszuleuchten. Alsdann ergibt sich auf einer eher nationalen Ebene insbesondere die Frage, welchen Einfluss MNU auf das Institutionengefüge verschiedener Nationalstaaten nehmen. Diese Frage erfreut sich nicht zuletzt aufgrund der wachsenden öffentlichen Anfragen an die Rolle von MNU in (Entwicklungs-) Ländern mit relativ schwach ausgebildeten Institutionen zunehmender Beliebtheit. Andererseits weist die zwar wachsende, aber zweifelsfrei noch vergleichsweise dünne empirische Forschung zum Einfluss von NBS auf MNU - auf Heimat- und Gastlandebene - noch erhebliche Lücken auf. Und schließlich bietet sich aus institutionalistischer Perspektive eine globale Ebene als immer drängenderes Forschungsgebiet an. Die rapide Entstehung und Weiterentwicklung globaler Institutionen - von Regierungsorganisationen wie der EU oder der WTO über globale Nicht-Regierungsorganisationen bis hin zu einer Vielzahl kleinerer Organisationen hat zunehmend direkten Einfluss auf unternehmerisches Handeln, speziell für global agierende MNU. Nicht nur die Entstehung und Gestaltung globaler Organisationen, sondern auch deren Einfluss auf unternehmerisches Handeln ist ein Forschungsfeld, welches gerade aus institutionalistischer Perspektive überaus spannend ist.

\section{Literatur}


Bartlett, Christopher/Sumantra Ghoshal (1989): Managing across borders: The transnational solution. 2 ed. Boston: Harvard Business School Press.

Bartlett, Christopher/Sumantra Ghoshal (1997): The transnational organization. In: D S Pugh (Hrsg.), Organization theory: selected readings. London: Penguin, S. 6482.

Becker-Ritterspach, Florian/Knut Lange/Karin Lohr (2002): Control Mechanisms and Patterns of Reorganization in MNCS. In: Mike Geppert/Dirk Matten/Karen Williams (Hrsg.), Challenges for European management in a global context. Basingstoke: Palgrave, S. 68-95.

Belanger, Jaques/Chritian Berggren/Torsten Björkman/Christoph Köhler (1999): Being local worldwide: ABB and the challenge of global management Ithaca: Cornell University Press.

Bergedorfer Gesprächskreis (2000): Modell Deutschland: Reif für die Globalisierung? Hamburg: Körber-Stiftung.

Berger, Peter L./Thomas Luckmann (1980): Die gesellschaftliche Konstruktion der Wirklichkeit: Eine Theorie der Wissenssoziologie Frankfurt am Main: Fischer.

Birkinshaw, Julian/Neil Fry (1998): Subsidiary to develop new markets. In: Sloan Management Review 39, S. 51-61.

Birkinshaw, Julian/Neil Hood (1998): Multinational subsidiary evolution: Capabilities and charter change in foreign-owned companies. In: Academy of Management Review 23(4), S. 773-795.

Birkinshaw, Julian/Neil Hood (2001): Unleash innovation in foreign subsidiaries. In: Harvard Business Review 79(3), S. 131-137.

Brunsson, Nils (1985): The irrational organization New York: John Wiley.

Buckley, Peter J (1996): The role of management in international business theory: A meta-analysis and integration of the literature on international business and international management. In: Management International Review 36(Special Issue), S. 111-137.

Buckley, Peter J/Pervez N Ghauri (Hrsg.) (1999): The Internationalization of the firm. A reader London: International Thomson Business Press.

Casson, Mark (1997): Information and Organisation: A new perspective on the theory of the firm Oxford: Clarenden Press.

Child, John/Alfred Kieser (1979): Organization and managerial roles in British and West German companies: An examination of the culture-free thesis. In: Cornelius 
J. Lammers/David J. Hickson (Hrsg.), Organizations alike and unlike: International and inter-institutional studies in the sociology of organizations. London: Routledge, S. 251-271.

Child, John (1981): Culture, contingency, and capitalism in the cross-national study of organizations. In: Barry M Staw/Larry L Cummings (Hrsg.), Research in organizational behaviour. Vol. 3. Greenwich/CT: JAI Press, S. 303-356.

Child, John (2000): Theorizing about organizations cross-nationally. In: Joseph L C Cheng/Richard B Peterson (Hrsg.), Advances in international comparative management, Volume 13. Stamford/CN: JAI Press, S. 27-75.

Child, John/David Faulkner/Robert Pitkethly (2001): The management of international acquisitions Oxford: Oxford University Press.

DiMaggio, Paul J/Walter W Powell (1983): The iron cage revisited: Institutional isomorphism and collective rationality in organizational fields. In: American Sociological Review 48, S. 147-160.

Djelic, Marie-Laure/Jabril Bensedrine (2001): Globalization and its limits: The making of international regulation. In: Richard Whitley (Hrsg.), The multinational firm. Oxford: Oxford University Press, S. 253-280.

Djelic, Marie-Laure/Sigrid Quack: 2002. From national configuration to transnational recombination. Paper presented at the 18th EGOS Colloquium, Barcelona, Spain.

Djelic, Marie-Laure/Sigrid Quack (Hrsg.) (2003): Globalization and institutions Cheltenham: Edward Elgar.

Edwards, Tony/Anthony Ferner: 2001 'Wall Street, 'short-termism' and the management of labour in American multinationals', ESRC Transnational Communities Programme Conference. Warwick.

Ferner, Anthony/Javier Quintanilla (1998): Multinationals, national business systems and HRM: The enduring influence of national identity or a process of 'AngloSaxonization'. In: Journal of Human Resource Management 9(4), S. 710-731.

Ferner, Anthony (2000): The underpinnings of 'bureaucratic' control systems: HRM in European Multinationals. In: Journal of Management Studies 37(4), S. 521-539.

Ferner, Anthony. (1997): Country of origin effects and HRM in multinational companies. In: Human Resource Management Journal 7(1), S. 19-37.

Financial Times: 2003. 'FT Special Report Global 500', Financial Times, 28/05/2003. 
Geppert, Mike: (2003). Sensemaking and politics in MNCs: A comparative analysis of vocabularies within the global manufacturing discourse in one industrial sector. In: Journal of Management Inquiry 12(4), S. 312-329.

Geppert, Mike/Dirk Matten/Karen Williams (Hrsg.) (2002): Challenges for European managment in a global context. Experiences from Britain and Germany Basingstoke: Palgrave.

Geppert, Mike/Ed Clark (2003): Knowledge and learning in transnational ventures:an actor-centred approach. In: Management Decision 41(5), S. 433-442.

Geppert, Mike/Dirk Matten/Karen Williams (2003): Change Management in MNCs: How Global Convergence Intertwines with National Diversities. In: Human Relations 56(7), S. 807-838.

Geppert, Mike/Karen Williams/Dirk Matten (2003): The social construction of contextual rationalities in MNCs: An Anglo-German comparison of subsidiary choice. In: Journal of Management Studies 40(3), S. 617-641.

Ghoshal, Sumantra/Christopher Bartlett (1993): The multinational corporation as an international network. In: S Ghoshal/D. Eleanor Westney (Hrsg.), Organization Theory and the multinational corporation. Basingstoke: Macmillan Press, S. 77104.

Granovetter, Mark (1992): Economic action and social structure: The problem of embeddedness. In: M. Granovetter/R. Swedber (Hrsg.), Sociology of economic life. Boulder: Westview Press.

Gunnigle, Patrick/Kevin R. Murphy/Jeanette N. Cleveland/Noreen Heraty/Michael Morley (2002): Localization in Human Resource Management: Comparing American and European Corporations. In: Michael A. Hitt/Joseph L C Cheng (Hrsg.), Managing transnational firms: Resources, market entry and strategic alliances. Amsterdam: JAI Press.

Hampden-Turner, Charles/Alfons Trompenaars (1993): The seven cultures of capitalism : value systems for creating wealth in the United States, Japan, Germany, France, Britain, Sweden, and the Netherlands. 1st ed. New York: Currency/Doubleday.

Hampden-Turner, Charles/Alfons Trompenaars (2000): Building cross-cultural competence : how to create wealth from conflicting values New Haven, Conn.: Yale University Press.

Harzing, Anne-Will/Arndt Sorge (2003): The relative impact of country of origin and universal contingencies on internationalization strategies and corporate control in 
multinational enterprises: worldwide and European perspectives. In: Organization Studies 24(2), S. 187-214.

Hedlund, Gunnar (1986): The hypermodern MNC: A heterarchy? In: Human Resource Management 25(9-36).

Hofstede, Geert (1994): Cultures and organizations. Software of the mind London: HarperCollins.

Hofstede, Geert (2001): Culture's consequences : comparing values, behaviors, institutions, and organizations across nations. 2nd ed. Thousand Oaks, Calif.: Sage.

Hollingsworth, J. Rogers/Robert Boyer (1997): Coordination of economic actors and social systems of production. In: J. Rogers Hollingsworth/Robert Boyer (Hrsg.), Comtemporary capitalism: the embeddedness of institutions. Cambrigde: Cambridge University Press, S. 1-47.

Lane, Christel (1992): European business systems: Britain and Germany compared. In: Richard Whitley (Hrsg.), European business systems. London: Sage, S. 64-97. Lane, Christel (1994): Industrial order and the transformation of industrial relations: Britain, Germany and France. In: R. Hyman/A. Ferner (Hrsg.), New frontiers in European industrial relations. Oxford: Blackwell, S. 167-195.

Lane, Christel (2000): Understanding the globalization strategies of German and British multinational companies. In: Marc Maurice/Arndt Sorge (Hrsg.), Embedding organizations: societal analysis of actors, organizations, and socioeconomic content. Amsterdam/Philadelphia: John Benjamins, S. 188-208.

Lane, Christel (2001): The emergence of German transnational companies: a theoretical analysis and empirical study of the globalization process. In: Glenn Morgan/Peer Hull Kristensen/Richard Whitley (Hrsg.), The multinational firm. Oxford: Oxford University Press, S. 69-96.

Levitt, T. (1983): The globalization of markets. In: Harvard Business Review 61(3), S. 92-102.

Lilja, Kari/Keijo Räsänen/Risto Tainio (1992): A dominant business recipe: the forest sector in Finland. In: Richard Whitley (Hrsg.), European business systems. London: Sage, S. 137-154.

Macharzina, Klaus/Michael-Jörg Oesterle/Dietmar Brodel (2001): Learning in multinationals. In: M. Dierkes/A. Berthoin Antal/John Child/I. Nonaka (Hrsg.), Handbook of organizational learning and knowledge. Oxford: Oxford University Press, S. 631-656. 
Matten, Dirk/Mike Geppert: 2002. Institutional Foundations of Manufacturing Strategies in Multinational Corporations: Evidence from Britain and Germany. Paper presented at the 18th EGOS Colloquium, Standing Working Group 1: Comparative Study of Economic Organizations, Barcelona.

Matten, Dirk/Mike Geppert (2004): Work systems in heavy engineering: the role of national culture and national institutions in multinational corporations. In: Journal of International Management 10(2), S. 177-198.

Maurice, Marc (1980): Societal differences in organizing manufacturing units: A comparison of France, West Germany and Great Britain. In: Organization Studies 1(1), S. 59-86.

Maurice, Marc/Arndt Sorge (Hrsg.) (2000): Embedding Organizations Amsterdam: John Benjamins.

McSweeney, Brendan (2002): Hofstede's model of national cultural difference and their consequences. In: Human Relations 55(1), S. 5-34.

Meyer, John W/Brian Rowan (1977): Institutionalized organizations. In: American Journal of Sociology 83, S. 340-363.

Meyer, John W. (2000): Globalization - Sources and Effects on National States and Societies. In: International Sociology 15, S. 233-248.

Morgan, Glenn (2001): The multinational firm: organizing across institutional and national divides. In: Glenn Morgan/Peer Hull Kristensen/Richard Whitley (Hrsg.), The multinational firm. Oxford: Oxford University Press, S. 1-24.

Morgan, Glenn/Peer Hull Kristensen/Richard Whitley (Hrsg.) (2001): The multinational firm Oxford: Oxford University Press.

Morgan, Glenn (2001b): Transnational communities and business systems. In: Global Networks 1(2), S. 113-130.

Nohria, Nitin/Sumantra Ghoshal (1997): The differentiated network. Organizing multinational corporations for value creation San Francisco: Jossey-Bass.

Porter, Michael (1980): Competitive strategy: Techniques for Analysing industries and competitors New York: Free Press.

Pries, Ludger (2001): The approach of transnational social spaces: Responding to new configurations of the social and the spatial. In: Ludger Pries (Hrsg.), New Transnational Social Spaces. London and New York: Routledge, S. 3-33.

Quack, Sigrid/Glenn Morgan/Richard Whitley (Hrsg.) (1999): National Capitalisms, Global Competition, and Economic Performance Amsterdam/Philadelphia: John Benjamins Publishing Company. 
Rosenzweig, Philip M./Jitendra V. Singh (1991): Organizational environment and the multinational enterprise. In: Academy of Management Review 16(2), S. 340-361.

Rugman, Alan M. (2000): The end of globalisation London: Random House.

Schmidt, Peggy. 2001. Nationalkulturelle und institutionelle Einflüsse in multinationalen Unternehmen: eine britisch - deutsche Fallstudie. Diplomarbeit, Humboldt-Universität, Berlin.

Scott, Richard W. (1998): Organizations: Rational, natural and open systems. 4th ed. Upper Saddle River, NJ: Prentice Hall.

Selznick, Philip (1949): TVA and the grass roots Berkeley: University of California Press.

Sorge, Arndt/M. Warner (1986): Comparative factory organisation. An AngloGerman comparison of management and manpower manufacturing Aldershot: Gower.

Sorge, Arndt/Wolfgang Streeck (1988): Industrial relations and technical change: The case for an extended perspective. In: R. Hyman/Wolfgang Streeck (Hrsg.), New technology and industrial relations. Oxford: Blackwell, S. 19-47.

Sorge, Arndt (1989): Organisationskulturen: Realer Hintergrund und soziologische Bedeutung einer Modewelle. In: Max Haller, Hoffmann-Nowotny, Hans-Joachim, Zapf, Wolfgang (Hrsg.), Kultur und Gesellschaft. Frankfurt: Campus Verlag, S. 193-209.

Sorge, Arndt (1991): Strategic fit and societal effect - interpreting cross-national comparisons of technology, organization and human resources. In: Organization Studies 12(2), S. 161-190.

Sorge, Arndt. (1995): Cross-national differences in personnel and organization. In: Anne-Will Harzing/Johann van Ruysseveldt (Hrsg.), International Human Resource Management. An integrated approach. London: Sage, S. 99-123.

Tainio, Risto/M Huolman/M Pulkkinen (2001): The internationalization of capital markets: how international institutional investors are restructuring finnish companies. In: Glenn Morgan/Peer Hull Kristensen/Richard Whitley (Hrsg.), The multinational firm. Oxford: Oxford University Press, S. 153-171.

Tempel, Anne/Peter Walgenbach: 2003. Global standardization of organisational forms and management practices?: Combining American and European institutionalism. Paper presented at the 3rd Conference of the European Academy of Management, Milan. 
Walgenbach, Peter (2000): Die normgerechte Organisation Stuttgart: SchäfferPoeschel.

Weber, Max (1905): Die protestantische Ethik und der "Geist" des Kapitalismus Tübingen: Archiv für Sozialwissenschaft und Sozialpolitik.

Westney, D. Eleanor (1993): Institutionalization theory and the multinational corporation. In: S Ghoshal/D. Eleanor Westney (Hrsg.), Organization Theory and the multinational corporation. Basingstoke: Macmillan Press, S. 53-76.

Westney, D. Eleanor/Srilata Zaheer (2001): The multinational enterprise as an organization. In: A. M. Rugman/Thomas Brewer (Hrsg.), The Oxford handbook of international business. Oxford: Oxford University Press, S. 349-379.

Whitley, Richard (Hrsg.): (1992): European business systems London: Sage.

Whitley, Richard/Peer Hull Kristensen (Hrsg.) (1996): The changing European firm London: Routledge.

Whitley, Richard (1997): Business systems. In: Arndt Sorge/M. Warner (Hrsg.), The IEBM handbook of organizational behaviour. London: International Thomson Business Press, S. 173-186.

Whitley, Richard/Peer Hull Kristensen (1997): Governance at Work. Oxford: Oxford University Press.

Whitley, Richard (1999): Divergent capitalisms. The social structuring and change of business systems Oxford: Oxford University Press.

Woywode, Michael (2002): Global Management Concepts and Local Adaptations: Working Groups in the French and German Manufacturing Industry. In: Organization Studies 23(4), S. 497-524.

$\mathrm{Xu}$, Dean/Oded Shenkar (2002): Institutional Distance and the multinational enterprise. In: Academy of Management Review 27(4), S. 608-618. 


\section{Biographische Notiz}

Mike Geppert

Geb. 1964. Senior Lecturer für Business Management am Centre for Business

Management des Queen Mary College der University of London, Großbritannien.

Arbeitsgebiete: Organisationstheorie, Organisationswandel und -lernen,

Institutionalismus sowie international vergleichende Managementforschung.

Dirk Matten

Geb. 1965. Principal Research Fellow am International Centre for Corporate Social Responsibility der Nottingham University Business School, Großbritannien, sowie Habilitand am Lehrstuhl für Produktionswirtschaft und Umweltökonomie an der Heinrich-Heine Universität Düsseldorf. Arbeitsgebiete: Internationales Management, Unternehmensethik, Umweltmanagement und Corporate Social Responsibility.

Peggy Schmidt

Geb. 1971. Project Development Officer in der Strategic Policy Unit der Walisischen Landesregierung in Cardiff, Großbritannien. Arbeitsgebiete: Europäische Strukturfonds, Entscheidungsprozesse in der EU; Individualisierung, Modernisierung, Globalisierung, Internationales Management; Industrielle Beziehungen und Organisationskultur. 


\section{English Title:}

The contribution of institutional theories for the understanding of organization and management processes in multinational corporations

\section{Zusammenfassung}

Der Beitrag arbeitet die jüngere anglo-amerikanische Diskussion im Bereich des International Managements auf und stellt Grundlagen, wesentliche Ansätze, Kernaussagen und Konsequenzen des europäischen und amerikanischen Institutionalismus dar. Insbesondere skizziert und evaluiert der Beitrag die Konsequenzen dieser Ansätze für die Theorie der multinationalen Unternehmung (MNU). Dabei wird das Erklärungspotential der Ansätze an vier verschieden Aspekten diskutiert: Der Rolle des evolutionären Paradigmas, dem Einfluss des Heimatlandes, der Macht und Autonomie von Tochtergesellschaften, sowie der Entstehung transnationaler sozialer Räume. Insbesondere wird diskutiert, an welchen Stellen institutionalistische Ansätze einen eigenen Beitrag zur Theorie der multinationalen Unternehmung leisten.

\section{Summary - Mike Geppert/Dirk Matten/Peggy Schmidt}

This paper provides an overview over one of the most recent debates in international management by discussing key assumptions, major approaches, main contributions and implications of European and American institutionalist theories. In particular the article discusses and evaluates the consequences of these approaches for the theory of the multinational firm. The potential of these approaches is explored by highlighting four areas of interest: the role of the evolutionary paradigm, the influence of the country of origin, power relations in and autonomy of subsidiaries and the emergence of transnational social space. In particular the argument focuses on how those institutionalist approaches may enrich our understanding of and theoretical insights into the multinational firm. 


\section{Anmerkungen}

1 Vgl. (Child, 2000) der zwischen "low" and "high context” Ansätzen in der international vergleichenden Managementforschung unterscheidet. Letztere betonen, den Einfluß sozialer kultureller und politischer Kontexte fuer das Verständnis von Entscheideungsprozessen in MNU. Erstere abstahieren davon und gehen davon aus, daß sich Struktur und Handlungen in MNU durch ökonomische, psychologische und technologische Universalismen immer mehr angleichen.

2 Djelic und Bensedrine unterscheiden in diesem Zusammenhang zwischen dem phänomenologischen, hauptsächlich in den USA entwickelten, und dem in der europäischen Managementvergleichsforschung entwickelten historischen Institutionalismus (Djelic/Bensedrine, 2001).

3 So der Titel des Standardwerkes zum NBS-Ansatz von Whitley (1999).

4 So zeigt z.B. ein kürzlich in der Financial Times publizierter Report, dass unter den ersten 10 der 500 (nach Marktkapitalisierung) am meisten globalisierten Firmen 9 aus den USA kommen. Unter den ersten 20 sind es 14 US Firmen und unter den ersten 50 immerhin noch 33. Der Report zeigt allerdings deutlich, dass neben den „drop outs“ vor allem durch den Kurseinsturz in den Aktienmärkten, MNU aus neuen Herkunftsländern Eingang in die Liste der 500 Global fanden. Hier wäre z.B. Russland zu nennen, dass erst 1998 in die Liste aufgenommen wurde, vgl. (Financial Times, 2003).

5 Siehe auch Tempel und Walgenbach (2003), die eine gute Übersicht der Gemeinsamkeiten und Unterschiede der beiden Institutionalismen geben.

6 In diesem Falle ist das ein positives Beispiel. Die Sechstage Arbeitswoche reduzierte sich für die Arbeitnehmer auf 5 Tage. Allerdings gibt es genügend Bespiele, wo Landesregierungen von MNU unter Druck gesetzt werden, wie es z.B. bei Auslandsdirektinvestitionen in Ost- und Mitteleuropa der Fall war, um z.B. Umweltgesetze oder Sozialgesetze zu ihren Gunsten und damit zuungunsten der Arbeitnehmer zu verändern. 